\section{Exploratory study: the COVID-19 pandemic and community-based animal organisations and households in the USA}

Peer Reviewed

\section{Dr Sarah DeYoung ${ }^{1}$} Dr Ashley Farmer ${ }^{2}$

1. University of Delaware, Delaware, United States of America.

2. Illinois State University, Illinois, United States of America.

\section{SUBMITTED}

30 March 202

\section{ACCEPTED}

7 May 2021

\section{DOI}

www.doi.org/10.47389.36.3.85

\section{(c) 109}

(c) 2021 by the authors. icense Australian Institute for Disaster Resilience, Melbourne Australia This is an open access article distributed under the terms and conditions of the Creative Commons Attribution (CC BY) license (https:// creativecommons.org/ icenses/by/4.0/).

\section{Introduction}

In the United States of America (USA), over 546,000 have people died of COVID-19 (Centers for Disease Control and Prevention 2021a), unemployment dramatically increased (Power 2020) and households and organisations faced new challenges associated with social and physical distancing and other protocols that were necessary to slow the spread of the virus. When people are affected by disasters and crises, animals can also experience adverse outcomes (Irvine 2009). Several studies on the COVID-19 pandemic and human-anima outcomes indicate some positive trends in companion animal adoption and fostering (Syzdlowski \& Gragg 2020). Other studies highlighted issues related to zoonotic disease transfer and risk from people to animals and vice versa (McNamara, Richt \& Glickman 2020). At the organisational level, animal shelters reported record-breaking animal adoptions and fostering during the spring of 2020 (Sydlowski \& Gragg 2020). Speculations exist that the sudden uptick in people working from home caused the increase in fostering and adoption because people felt they had extra time to devote to animals and as a way to cope with the stress they experienced during the pandemic (Bussolari et al. 2021). Other studies show that bonding between people and their companion animals increased during pandemic lockdowns (Kogan et al. 2021a).

There are complex effects for companion animals with shifts of work and life patterns. Early research on dogs in the United Kingdom suggests that daily activities for dogs such as walking outside have been reduced (Christley et al. 2021). In addition, owners expressed concerns about limited veterinary visits and other physical changes due to lockdowns (Kogan et al. 2021a, Ratschen et al. 2020) and specific concerns about the affordability of care for companion animals (Kogan et al. 2021b). Bowen and co-authors (2020) suggest that, in addition to human stress that increased during the pandemic, companion animals may also display signs of increased stress.

While the positive aspects of animal services have been broadened by mainstream media (such as increased

\section{Abstract}

The COVID-19 pandemic has changed many aspects of human systems. Gaps in community services for people with companion animals can prevent people from seeking care during a pandemic or create other issues. This paper describes exploratory research to identify some key challenges and successes for animal services providers and for households with companion animals. Using data from 19 USA states were gathered using an online survey and respondents were from 13 animal services organisations and 90 households. Themes were identified based on organisationallevel challenges or successes, as well as themes at the household level. These findings may be useful for emergency managers and planners who design outreach and support services for people with companion animals, for example, planning for low-cost animal boarding services for people hospitalised or unable to care for their animal. 
fostering and adoption), this growing body of initial research is showing that issues (i.e. cost of veterinary care) will become prevalent as possible results of unemployment and disparities in wealth and health. Based on work on animals and social systems in disasters (DeYoung \& Farmer, in press), the changes in the consequences for animals will not be uniform. This is examined at 2 main levels: the organisational level and the household level.

\section{Rationale}

This exploratory study serves as the basis for future research on the ways in which animal service organisations and households with companion animals are effected by pandemics. It is critical to identify the challenges that organisations and households face to identify ways to improve planning and preparedness. An issue raised was that in guidelines provided by the Centers for Disease Control (2021b), people were encouraged to find alternatives for caring for their companion animals if they or someone in the household contracted COVID-19. However, the guidance on how to specifically navigate this process was hard to find. Additionally, people may make decisions about seeking care or other behaviours during the pandemic because of their attachment to their companion animals. These decisions can influence their appropriate responses (e.g. timely evacuation to a shelter or relocation of animals to suitable holding places before a hazardous event). Human-animal attachments can influence decision-making (Hosey \& Melfi 2014), including in disasters (Thompson 2013).

For animal services organisations, they indicate resilience to the pandemic depending on their ability to 'leverage' the situation to increase adoption or fostering events. This would be consistent with research by (DeYoung \& Farmer, in press) covering multiple disasters in which some organisations were better at capturing media attention, mobilising volunteers and conducting during and after disaster or hazard events. Organisations that can adapt during disasters may have better outcomes (Linnenluecke, Griffiths \& Winn 2012) and animal services organisation were most likely to experience this during the pandemic. However, it is unclear what specific mechanisms or organisational characteristics facilitate this adaptivity. This study explored some aspects of organisational resilience during the pandemic.

This study also considered the barriers in access to resources that lower-income households experience and the challenges related to companion animal care. One way to measure this is to identify the relationship between household income and actual adoption and fostering rates. Specifically, the hypothesis is that higherincomes households will be more likely to adopt a pet as well as foster new animals because they have additional resources to care for animals. In addition, because people view companion animals as pets, the study might show evidence of companion animals being buffers against stress during the pandemic.

\section{Method}

This research was approved by the University of Delaware Institutional Review Board (approval number 1693678-1). Data collection was carried out between January and February 2021.
A systematic social media recruitment approach in which a detailed list of organisations is created in spreadsheet for recruitment in groups that are specific to the topic of research (DeYoung \& Mangum 2021, Mongold et al. 2020) was used to gather responses from people in the USA regarding their perceptions of issues related to companion animals and animal services organisations during the COVID-19 pandemic. A systematic list of animal-focused groups covering broad geographic regions was used to recruit people to take the survey. Many of the groups recruited focused on general animal issues (lost and found) or animals and disasters. Two main groups of people were targeted:

- people who work or volunteer in animal services organisations - households with companion animals.

A Qualtrics (Provo, UT) survey contained 15 items: 11 items for household respondents and 4 additional items for respondents from organisations. There were also 3 open-ended questions and all respondents had the option to respond to the final question 'What else would you like to share?'. The questions included demographics (income, state of residence, ethnic background) and Likert-scale questions. For example:

Please indicate your agreement with the following statements, with 1 being the least amount of agreement and 5 being the highest level of agreement. 'My organisation has had to change internal operations and protocols because of COVID-19.'

(Question specifically for organisational respondents, 5 point disagree to agree)

\section{Regarding your HOUSEHOLD, please indicate the following: \\ 'I needed someone to care for my animal when someone in my household had COVID-19 but was unable to find someone.'}

(Question specifically for household respondents, yes/no response).

The average time a respondent spent completing the survey was 5 minutes after filtering for 'false' responses (people who clicked on the survey and then immediately closed out of it). Numeric data were analysed using Statistical Package for Social Sciences (SPSS) and open-ended items were coded in Excel using a content-analysis approach (Miles, Huberman \& Saldaña 2018).

\section{Results}

Before filtering and cleaning data based on response time, there were 107 survey responses. If respondents indicated 'no' to agreeing to participate or if they did not complete the survey beyond 1 survey item, the response was excluded from analysis. Respondents were mostly Caucasian with varied household incomes (Figure 1). Eleven respondents were from organisations (1 from Pennsylvania, 3 from California, 1 from Colorado, 1 from Florida, 1 from Delaware, 4 not listed) and 90 were from households, for a total of 101 respondents. 
Respondents came from 19 USA states as well as 1 respondent from Puerto Rico. Respondents were from California ( $n=9)$, Illinois $(n=9)$, Pennsylvania $(n=9)$, Delaware $(n=7)$, Kentucky $(n=4)$, Maryland $(n=3)$, North Carolina $(n=3)$, Florida $(n=3)$ and Georgia $(n=3)$. Approximately 46 per cent $(n=50)$ of household respondents indicated they adopted a new companion animal during the pandemic and 20 per cent $(n=22)$ of household respondents indicated they fostered a companion animal during the pandemic.

To test for relationship between income and fostering an animal, a Chi-square analyses was conducted on respondent income. Specifically, the categories between 'Less than $\$ 10,000$ ' and ' $\$ 50,000-\$ 59,000$ ' were recoded as 0 and categories for $\$ 60,000$ or higher were recorded as 1 (see Figure 1). The Chi-squares compared the recoded income with indications of adopting or fostering a new pet. There were no significant findings for either comparison:

- for adoption and income, $X^{2}=2.599$ (1), $p=0.107$

- for fostering and income, $X^{2}=0.008$ (1), $p=0.929$.

The scope and size of animal services organisations varied and included clinical veterinary services and fostering and adoption networks. They ranged in organisational type from limited liability companies and private organisations for profit to small and large-scale not-for-profit agencies.

The final open-ended question was independently coded and consensus was established on first- and second-round codes for themes (Saldaña 2014). There were 7 themes identified; 2 across organisations and 5 for households. The organisational themes corresponded with items asking about challenges and successes, while the remaining 5 codes came from the open-ended item that had a variety of responses about the pandemic experience. To check for inter-rater reliability of the open-ended data of the survey question, 'What else would you like to share with us regarding pets, animals, and the COVID-19 pandemic? an intraclass correlation analysis was conducted in SPSS and the Kappa score among the 2 analysists as raters was $\mathrm{K}=0.74$.

The 5 core household themes identified were 'owner financial concerns', 'owner wellbeing', 'animal behaviour', 'fostering/ adoption issues' and 'other'. The 'other' responses included general comments about the pandemic, observations about changes in veterinary protocols and comments such as,

'Household pets are great, but don't forget to feed feral cats and provide them shelter if possible'.

\section{Organisational themes}

Respondents indicated that fundraising had been negatively affected by the pandemic. This was connected to the restrictions in conducting fundraising events that would normally be held face-to-face. For example, one respondent noted:

With the inability to do in-person fundraising, we only brought in about half in 2020 of what we did in 2019. Our big in-person fundraiser, which in 2019 raised \$20,000, this year raised $\$ 7,000$.

The inability to host face-to-face events spilled over into fostering and adoption. One respondent indicated that not being able to run adoption events or fundraisers was a challenge.

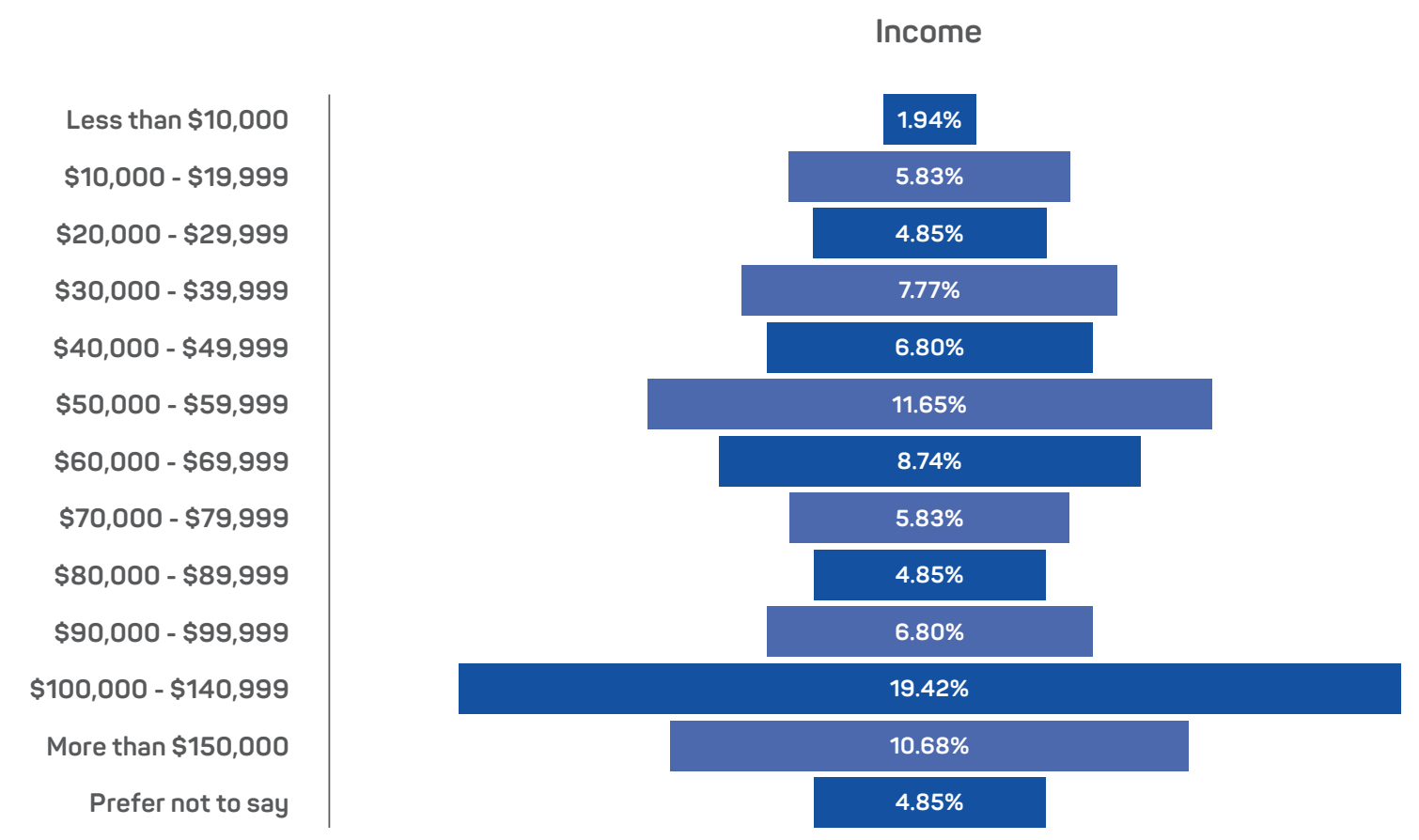

Figure 1: Percentage of respondents within each income category used in the survey. 
Another problem that organisational respondents indicated was appropriate vetting of people who wanted to adopt an animal during the pandemic but who perhaps did not have adequate resources or time to care for the animal. One respondent noted 'COVID adopters' as a challenge:

Screening adopters that may have good intentions but fall into the category of being a 'COVID adopter', which will result in a return of [the] adopted dog.

Respondents indicated that the physical location and processes for service delivery of their organisation were resilient if they were already outdoor-based activities:

Because TNR ${ }^{1}$ is a fairly solitary endeavour, and our trap pick-up and training sessions have always been held outdoors, there wasn't much change. Even when taking cats to barn homes it was easy to socially distance and stay outdoors.

This comment indicates that the organisation had a system in place that was conducive to the social distancing requirements because trap pick-up and training was already conducted outdoors. This meant that the volunteers and project leaders did not have to make alternative plans and design new processes for their operations.

While some respondents from organisations indicated negative outcomes, other respondents indicated that their organisation was able to adapt in some way. However, they experienced challenges due to staffing and burnout:

Our animal hospital quickly adjusted to not allowing clients in buildings. We meet clients at their cars and discuss their pet's visit over the phone. Our biggest issue has been an overworked staff.

This demonstrates that veterinary services and other organisations adapted to the 'kerbside' model of care, but staff experienced fatigue and burnout from working long hours. On a positive note, a respondent indicated that the shift to 'kerbside' care improved the workplace environment for people at their workplace:

I think our vets have enjoyed the fact that the clients haven't been in the building! Our staff has pulled together...it's the best group we have ever had. With folks not being in the building we have had the opportunity to address a lot of issues that were plaguing our old building without disrupting business operations.

This idea of 'pulling together' is reflective of the sense of community that veterinary staff may have experienced as the pandemic continued. While they felt stressed because of the general state of the world, work-life challenges and other issues, they worked together to achieve the goal of continuity of care for their clients. Another respondent indicated:

While most businesses were struggling and there were many negative impacts of COVID, overall it had a positive outcome for us. People found they had more time to work with their foster dogs, we gained new foster families when most were homebound, we were able to take in many more difficult cases due to the uptick in foster involvement and donations.

This shows that the organisation was able to harness the new resources (people willing to foster dogs) to bolster the organisation's success and perhaps its visibility. While these positive factors were present in the data, it should be noted that respondents also indicated a sense of despair:

The pandemic made many animals homeless, by their owners having to give them up or by them passing away. Many more were born outside because TNR was suspended during the most crucial seasons. While many people adopted pets at the beginning of the pandemic, it did not make up for that.

In other words, some respondents were concerned about the effects that deaths from COVID-19 had on animals and the spillover consequences such as pet overpopulation due to the delay in spay and neuter services during the pandemic.

\section{Household themes}

\section{Financial concerns}

Responses reported that financial concerns were directly related to their capacity to seek care for animals during illness. For example, respondents indicated that their role as a caregiver for their companion animal prevented them from seeking timely medical care for themselves-even if they were in a lifethreatening situation:

I got COVID and delayed going to the emergency room because I couldn't find anyone to care for my fur kids. I waited until I networked with a group of friends and their friends to find several people to take my fur kids. I have 6 cats and a service dog. My fur kids are still in foster care 2 months later because I'm still sick, with the exception of 2 of my fur kids that have medical problems that were medically boarded and are home after being boarded for a month. They're only home because I couldn't afford the $\$ 100$ a day it costs to medically board them any longer and I couldn't find anyone who could foster them.

This highlights the need for expanded services for people without access to resources, extended family and social networks and other forms of support. This respondent revealed multiple layers of vulnerability (having a service dog and limited social networks of people who could care for their pets). This is a problem in disasters and pandemics and could be addressed through expanded services (such as federal incentives and subsidies for veterinary clinics to provide services on a sliding scale for households with extremely low income) and additional research on the vulnerabilities of households with companion animals.

1. Trap, neuter, return (usually a practice for sterilising feral cats). 
Another respondent described how their financial situation restricted their ability to continue with their pet's routine veterinary care:

Finances are tight, so I've used the stimulus money for vet care this past cheque.

This theme was not surprising because rates of joblessness associated with the pandemic rose during 2020. While many animal food pantries and human meal delivery services were designed as stop gaps to support people, the cost of veterinary care (especially emergency veterinary care) can be a significant burden for owners.

\section{Owner wellbeing}

Respondents indicated that their companion animals were a source of positive mental health coping during the pandemic. For example, respondents stated that the pandemic would not have been tolerable without their animals and that having an animal around created a sense of 'normalcy':

I moved to working at home 100 per cent of the time at the beginning of the pandemic and I would have probably had more issues with my mental health if I didn't have my dog and then the two kittens we adopted. They have been wonderful to have at home with me so I don't feel lonely all the time. I worry about them and how they will handle the separation once I do eventually return to some out-ofhome work.

Another respondent indicated:

My kitten has been the light of my life through COVID, especially since I live alone.

This suggests that people who live alone may have been more likely to adopt or foster a new companion animal during the pandemic. The motivation to adopt or foster might be partially context-specific and, in some ways, dependent on household characteristics. It is also possible that families with small children may have viewed the lockdowns as an ideal time to adopt an animal if it was something they were considering before the pandemic.

\section{Animal behaviour}

Respondents described how the pandemic was associated with reduced opportunities for animal socialisation because of social distancing and changes in the daily activities of owners:

Our pandemic rescue puppy, adopted in September at around 3 months old, is definitely under socialised! So much of the typical advice about how to raise a puppy does not account for quarantine/isolation practices, and we are expecting that as he grows up he may be a little more reactive or standoffish than he otherwise would have been.

Some respondents described anticipatory concerns about how their pets would cope with 'regular' daily routines after the pandemic:
Pre-pandemic, my dog had diagnosed separation anxiety. Knowing that my time working from home exclusively will eventually end, I am worried about the financial and mental costs for me to overcome his anxiety again.

Some respondents described their experiences in contracting COVID-19 and waiting to seek care. Other respondents described how animals improved their mental health, or that because of special or functional and access needs, the animal played an important role in their life during the pandemic.

There was evidence on social media and in the news of people not being able to find care for their animals if someone in the household had COVID-19. However, this trend did not show in the data. Only 1 respondent indicated 'yes' to the question 'I needed someone to care for my animal when someone in my household had COVID-19 but was unable to find someone'. Three respondents indicated that they allowed someone else outside of the household to care for their pet because someone within their household had COVID-19.

\section{Fostering and adoption}

Respondent comments reflected on ways in which the pandemic facilitated decision-making for new fostering or adoption. For example, one respondent described working from home as a major factor in deciding to bring a new companion animal into the household:

We finally adopted because our jobs moved to remote work, and we had the flexibility in our jobs to take on a new pet (periodic breaks to go outside, house training, fun training).

There were also some comments that reflected how organisational processes made adoption more difficult:

Adoption was a pain, we pretty much had to find an org that was arguably flouting the rules to even be able to meet any cats before adopting one.

This suggests that during the pandemic it may have been challenging for some adopters spend time with the dog or cat at the physical shelter to see if the animal was a good match for the household prior to adoption. There were also instances in which some people said they had adopted an animal specifically because of mental health needs associated with the pandemic:

I had to get an emotional support letter from my doctor in order to adopt a pet. I would not have gone through these steps had we not been in a pandemic.

This comment might also be indicative of new procedures that some shelters required to ensure that animals would not be immediately surrendered after the pandemic ended. This is important to note because while the media and news stories focused on increases in adoptions and fostering, there were complexities that were less visible to the public related to shifts in operational protocols that created challenges in adoption or fostering. 


\section{Discussion}

These findings indicate that:

- organisations did, in some way, have specific coping mechanisms for remaining operational and successful during COVID-19 (despite some challenges)

- households with companion animals had improved coping mechanisms for dealing with stress but that there were also concerns about finances and the impact on the socialisation and behaviour of their pet.

Respondents indicated a hesitation to seek medical care because of their companion animals and the logistics associated in finding boarding or care.

For organisations as well as for households, there was uncertainty about finances and this was associated with comments about stress. An hypothesis that households in higher-income categories would be more likely to adopt or foster was not evidenced. The Chi-square comparisons showed no significant effect in income and new adoptions or fostering. This does not necessarily mean that financial limitations did not have an effect on households. Respondents indicated that they worried about the cost of veterinary care. Additionally, while the Centers for Disease Control and Prevention recommended that people not have physical contact with their pet if they tested positive for COVID-19, it was not clear what people should do when they did not have others in their social network to care for their pets or if they could not afford boarding. One respondent raised this lack of government guidance or community care for people and animals during the pandemic:

As an occupational therapist in home health, I saw many homebound patients that had no awareness of best practices for pets in terms of need to have designated caregivers in case of hospitalisation, obtaining food and care services and precautionary disinfectant needs. No information from vets was provided to owners or to the community in general, therefore a lack of awareness.

The growth in numbers of 'COVID-19 adopters' is interesting because it indicates a pattern in which people may want to adopt 'hurricane dogs' for the 'brand' or image of doing so. It is difficult to assess the valence of this because an increase in adoptions during a crisis (such as a hurricane or a pandemic) can be a positive outcome for the organisation and for the animals. It is also possible that the respondent may be concerned that the household would be affected by COVID-19 and the animal surrendered. Future qualitative research should include questions to understand the perceptions associated with adopting and fostering 'pandemic animals'.

\section{Limitations}

There are several limitations to this study. The data were collected through purposive sampling that was focused on people who were already 'interested' or possibly enthusiastic about animal services and animal issues. The groups recruited were animal-centric and respondents may be more willing to adopt and foster animals than a member of the general population in the USA. Additionally, the respondents were mostly Caucasian and were in the middleto-upper income categories. This narrow diversity prevents making clear and meaningful interpretations about disparities in animal services organisations or household experiences with companion animals that might be related to other cultural, social or demographic factors (ethnic background, etc.). According to Bassett, Chen, and Krieger (2020), 'Black and Hispanic people' in the USA have experienced disproportionate deaths due to COVID-19. It is unclear to what extent this overlaps with issues related to companion animals. It should be noted that the respondent sample in the current study is representative of the population of people who own companion animals in the USA, since:

Pet ownership differs among racial and ethnic groups. The highest rate of pet ownership overall in 2016 was seen among White households (65 per cent), with Latino/ Hispanic (61 per cent) next. The lowest rate was found among Black/African American households (37 per cent).

(American Veterinary Medical Association 2018, p.5).

Another limitation is that the sample of 90 households and 11 animal care organisations is small, although it was designed to be exploratory and identify issues that should be examined.

Future research could explore if people who fostered animals during the pandemic were more likely to adopt the animal (also known as a 'foster failure'). DeYoung and Farmer (in press) found evidence that people may 'trauma bond' during disasters where people who normally fostered a higher number of animals that were normally subsequently adopted by other people, chose to keep the fostered animal because there was a sense of having lived through the crisis together. It would be interesting to see if this is the case for special populations during the pandemic such as older people in isolation, frontline health workers, children with special needs and other groups that might experience emotional benefits or bonding with companion animals.

\section{Conclusion}

The COVID-19 pandemic, like many emergency scenarios, affected both humans and animals. This study highlighted some of the changes and effects for organisations and households in the USA. While there were challenges with raising money, as well as financial difficulties at the household level, there were also positive outcomes, such as higher rates of animal adoptions and fostering and effective adaptation by animal services organisations to provide kerbside vet care. Many individuals turned to their companion animal for comfort and coping. Some respondents indicated a delay in accessing medical care while they were unwell because of difficulties finding affordable care for their companion animals.

While the long-term consequences of the pandemic are unknown, the human-animal relationship continues to be affected in a variety of ways by emergencies and hazards. Implications from these findings include new potential policy solutions that would support animal management organisations (not-for-profit and government-run sheltering organisations) to maintain continuity of service during pandemics. This might include funding that supports temporary shifts in operational 
protocols associated with social and physical distancing. Temporary support programs for veterinary clinical services can provide additional relief to small veterinary businesses.

Lessons for emergency management planning include clear mechanisms for providing low-cost care and safe boarding for companion animals for people in hospital due to COVID-19. This is similar to evacuation refusal, but more careful consideration for space, logistics and technical animal support services should be integrated in a mass care pandemic plan.

There may be benefits to households with companion animals if veterinary services and other organisations provided guidance on managing animal anxiety. While animals providing emotional support in crisis is not new, animal services organisations can leverage the 'pandemic recovery' timeframe to garner public support and private donations for adoption services and pet overpopulation programs. Event-leveraging has worked to bolster donations during past disasters. Recovery from this pandemic could be modelled on past disaster fundraising programs and efforts for animal service organisations.

\section{References}

American Veterinary Medical Association 2018, 2017-2018 AVMA Pet Ownership and Demographics Sourcebook. Schaumburg IL: American Veterinary Medical Association.

Bassett MT, Chen JT \& Krieger N 2020, Variation in racial/ethnic disparities in COVID-19 mortality by age in the United States: A cross-sectional study. PLoS medicine, vol. 17, no. 10, p.e1003402.

Bowen J, García E, Darder P, Argüelles J \& Fatjó J 2020, The effects of the Spanish COVID-19 lockdown on people, their pets, and the humananimal bond. Journal of Veterinary Behavior, vol. 40, pp.75-91.

Bussolari C, Currin-McCulloch J, Packman W, Kogan L \& Erdman P 2021, 'I Couldn't Have Asked for a Better Quarantine Partner!': Experiences with Companion Dogs during Covid-19. Animals, vol. 11, no. 2, p.330.

Centers for Disease Control and Prevention 2021a, COVID Data Tracker. At: https://covid.cdc.gov/covid-data-tracker/\#datatrackerhome.

Centers for Disease Control and Precention, 2021b, COVID-19 and Animals. At: www.cdc.gov/coronavirus/2019-ncov/daily-life-coping/ animals.html.

Christley RM, Murray JK, Anderson KL, Buckland EL, Casey RA, Harvey ND, Harris L, Holland KE, McMillan KM, Mead R \& OwczarczakGarstecka SC 2021, Impact of the First COVID-19 Lockdown on Management of Pet Dogs in the UK. Animals, vol. 11, no. 1, p.5.

DeYoung SE \& Farmer AK (in press), All creatures safe and sound: the social landscape of pets in disasters. Temple University Press, Philadelphia, PA.

DeYoung SE \& Mangum M 2021, Pregnancy, Birthing, and Postpartum Experiences During COVID-19 in the United States. Frontiers in Sociology, vol. 6, no. 12.

Hosey G \& Melfi V 2014, Human-animal interactions, relationships and bonds: A review and analysis of the literature. International Journal of Comparative Psychology.
Irvine L 2009, Filling the Ark. Temple University Press.

Kogan LR, Erdman P, Bussolari C, Currin-McCulloch J \& Packman W 2021a, The initial months of COVID-19: Dog owners' veterinaryrelated concerns. Frontiers in Veterinary Science, vol. 8, p.45.

Kogan LR, Erdman P, Currin-McCulloch J, Bussolari C \& Packman W 2021b, The Impact of COVID on Cat Guardians: Veterinary Issues. Animals, vol. 11, no. 3, p.603.

Linnenluecke MK, Griffiths A \& Winn M 2012, Extreme weather events and the critical importance of anticipatory adaptation and organizational resilience in responding to impacts. Business strategy and the Environment, vol. 21, no. 1, pp.17-32.

Miles MB, Huberman AM \& Saldaña J 2018, Qualitative data analysis: A methods sourcebook. Sage Publications.

Mongold E, Davidson RA, Trivedi J, DeYoung S, Wachtendorf T \& Anyidoho P 2020, Hurricane evacuation beliefs and behaviour of inland vs. coastal populations. Environmental Hazards, pp.1-19.

McNamara T, Richt JA \& Glickman L 2020, A critical needs assessment for research in companion animals and livestock following the pandemic of COVID-19 in humans. Vector-Borne and Zoonotic Diseases, vol. 20, no. 6, pp.393-405.

Power K 2020, The COVID-19 pandemic has increased the care burden of women and families. Sustainability: Science, Practice and Policy, vol. 16, no. 1, pp.67-73.

Qualtrics 2021, Provo UT, Version February 2021, At: www. qualtrics.com.

Ratschen E, Shoesmith E, Shahab L, Silva K, Kale D, Toner P, Reeve C \& Mills DS 2020, Human-animal relationships and interactions during the Covid-19 lockdown phase in the UK: Investigating links with mental health and loneliness. PloS one, vol. 15, no. 9, p.e0239397.

Saldaña J 2014, Coding and analysis strategies. In The Oxford handbook of qualitative research.

Szydlowski M \& Gragg C 2020, An Overview of the Current and Potential Effects of COVID-19 on US Animal Shelters. AIJR Preprints.

Thompson K 2013, Save me, save my dog: Increasing natural disaster preparedness and survival by addressing human-animal relationships. Australian Journal of Communication, vol. 40, no 1, pp.123-136.

\section{About the authors}

Dr Sarah DeYoung is at the Disaster Research Center at the University of Delaware, the Sociology and Criminal Justice Department and the Joseph R. Biden School of Public Policy and Administration. Her research focuses on protective action decision-making in disasters and social issues related to companion animals in disasters.

Dr Ashley K Farmer is an Assistant Professor in the Department of Criminal Justice Sciences at Illinois State University. Her areas of research are in policing, specifically police-community relations and how technology is used. 